\title{
Efficiency Analysis of Organic Light-Emitting Diodes based on Optical Simulation
}

\author{
Benjamin C. Krummacher, ${ }^{a, *}$ Stefan Nowy ${ }^{b}$, Jörg Frischeisen ${ }^{b}$, \\ Markus Klein a, Wolfgang Brütting ${ }^{b, \dagger}$
}

a) OSRAM Opto Semiconductors, OLED-Lighting, Leibnizstr. 4, D-93055 Regensburg, Germany

b) Experimental Physics IV, University of Augsburg, 86135 Augsburg, Germany

\begin{abstract}
In spite of huge progress in improving the internal quantum efficiency of organic light-emitting diodes (OLEDs), these devices still suffer from poor light out-coupling. Loss mechanisms are for example waveguiding in the organic layers and the substrate as well as the excitation of surface plasmons at metallic electrodes. Their relative strength and the mutual dependence on the OLED structure have been studied both experimentally and by numerical simulation. Here, we consider the impact of the radiative quantum efficiency of the emitter material on predictions of light extraction from OLEDs. Competing processes resulting in non-radiative recombination of charge carriers usually reduce the emitter quantum efficiency in a real device. We show that optical simulation leads to erroneous conclusions when neglecting these competing processes. Furthermore, we demonstrate a method, which allows determining both the radiative quantum efficiency and the charge recombination factor
\end{abstract}

\footnotetext{
Electronic address: benjamin.krummacher@osram-os.com

† Electronic address: wolfgang.bruetting@physik.uni-augsburg.de
} 
via simulation based analysis of experimental data. This analysis of device efficiency is applied on a set of red-emitting electrophosphorescent devices. 


\section{INTRODUCTION}

Organic solid state lighting technology is on the verge of creating commercial applications in the lighting sector. The possibility of producing a flat large area lighting panel drives the development of creative products and opens exciting fields of application. However, one evident drawback is still the low light out-coupling efficiency of organic lightemitting diodes (OLEDs). The external device efficiency is significantly reduced by various optical loss channels. Considering a conventional coplanar bottom-emitting OLED based on an ITO coated glass substrate a major part of the generated radiant power is lost to thin film waveguided modes (or ITO/organic waveguided modes), surface plasmon polariton modes of the metal cathode and substrate waveguided modes. Furthermore a small fraction of the generated radiation is absorbed by the materials of the different layers.

The modification of the substrate surface directed towards the ambient environment is a basic approach to increase external device efficiency. Diffuse layers (i.e. scattering particles in a transparent matrix [1-4]), microstructured films (e.g. microlens arrays [5]) or roughened substrates [6] are well-known techniques for this purpose. Application of a diffusor or sandblasting the substrate are low cost approaches which could be used in future mass production.

Although external device efficiency could be increased by such methods, substrate surface modification cannot be utilized, when a reflecting off-state appearance of the bottomemitting OLED is desired. The optimum configuration of such a device differs from the one of a device with substrate surface modification: Maximum light has to be directed into the light escape cone of the substrate in the case of a non modified substrate. On the other side maximum radiant power has to be coupled from the thin film stack into the substrate when using substrate surface modification. Here, we neglect aspects regarding the target emission color (e.g. there is a wavelength dependence of the enhancement factor of light outcoupling, 
which leads to a change in emission color after applying the substrate surface modification [7]).

Recently, we have studied optical loss mechanisms and the light extraction efficiencies of OLEDs by means of a dipole model [8]. We have shown that experimental data on the angular dependent emission spectra of green bottom-emitting OLEDs are in excellent agreement with optical simulations. Furthermore, we have demonstrated the influence of different device parameters, like organic layer thicknesses and the position of the emission layer, on the coupling of the emitting dipoles to different optical channels in an OLED. In the present article we consider the impact of the radiative quantum efficiency of the emitter material on predictions of external device efficiency obtained from optical simulation in more detail. The radiative quantum efficiency $q$ of an emitting dye is defined as the quantum efficiency in an unbounded space filled by the dye and its host. Non-radiative recombination of charge carriers, e.g. by bimolecular recombination processes or interaction with charge carriers, reduces the value of $q$. The intention of this paper is twofold: First we will demonstrate that optical simulations neglecting the impact of these competing processes on $q$ lead to erroneous results not only in terms of the total amount of light extracted from an OLED, but also concerning the optimal device architecture to achieve this. Secondly, we will show that even if the radiative quantum efficiency of an emitter material is not known beforehand, it can be extracted by an analysis of experimental data based on optical simulation.

\section{METHOD}

\subsection{Experimental}

The structure of the red-emitting electrophosphorescent OLEDs used for this study was $113 \mathrm{~nm}$ indium tin oxide (ITO) / $203 \mathrm{~nm}$ hole transporting layer (HTL) / $10 \mathrm{~nm}$ electron blocking layer (EBL) / $7 \mathrm{~nm}$ emission layer (EML) / $10 \mathrm{~nm}$ hole blocking layer (HBL) / 
electron transporting layer (ETL) / $200 \mathrm{~nm} \mathrm{Ag-cathode} \mathrm{(see} \mathrm{Fig.} \mathrm{1).} \mathrm{The} \mathrm{thickness} \mathrm{of} \mathrm{the} \mathrm{ETL}$ was varied in the range from $48 \mathrm{~nm}$ to $110 \mathrm{~nm}$. The peak wavelength of the photoluminescence spectrum of the red-emitting phosphorescent dye was $593 \mathrm{~nm}$.

The ETL and HTL were doped by materials improving electron and hole transport, respectively. The diodes were fabricated as follows: The organic stack was deposited on ITO coated glass substrates (refractive index 1.52) using standard evaporation technique from crucibles. The evaporation rate was $1 \AA / \mathrm{s}$ at a base pressure of $10^{-7} \mathrm{mbar}$. Following evaporation of the Ag-cathode, the devices were encapsulated with a glass lid and getter. The ETL and its dopant, the HTL and its dopant, and the matrix of the EML and its red dye were applied by co-evaporation. The active area of the OLEDs was $4 \mathrm{~mm}^{2}$. Additionally single layers were deposited on silicon substrates. To ensure accurate optical simulation, the complex index of refraction of the samples as a function of wavelength was determined by means of standard spectral ellipsometry for all organic layers.

The current density-voltage $(\mathrm{J}-\mathrm{V})$ characteristics of the devices used in this study were measured by means of a Keithley 238 as current source and a Keithley 6514 as voltmeter. The experimental $\mathrm{J}-\mathrm{V}$ characteristics of the devices with thinnest $(48 \mathrm{~nm})$ and thickest $(110 \mathrm{~nm})$ ETL are plotted in Fig. 2. Both characteristics do not differ significantly, which can be attributed to appropriate n-doping. As will be shown below, the optical properties of the device are strongly affected by the thickness variation of the n-doped ETL, while there is almost no change in the electrical properties of the diode.

In order to collect both directly emitted light and light coupled from the active layers into the substrate, the bottom emitting OLEDs with planar substrate ( $4 \mathrm{~mm}^{2}$ active area) was coupled to the center of a glass hemisphere (diameter $25 \mathrm{~mm}$, refractive index 1.52) by using a refractive index matching gel obtained from Cargille Laboratories. The configuration effectively makes the emission angle in glass equal to the emission angle in air. Thus nearly all photons hit the surface of the glass hemisphere at normal incidence. This allows the 
measurement of the OLED emission within the substrate at angles exceeding the critical angle of total internal reflection between glass and air $[9,10]$. The emission of the OLED equipped with the glass hemisphere was measured in an integrating sphere, which was connected to a fiber spectrometer (Instrument Systems CAS 140B). The Comission Internationale de 1’Éclairage (CIE) color coordinates related to the measured spectra of the different devices are given in Table 1. Additionally, the graph in Fig. 3 shows the external quantum efficiency as a function of current density as obtained from the measurements of the device with $72 \mathrm{~nm}$ ETL in the integrating sphere.

\subsection{Optical Modeling}

The optical modeling of OLEDs must consider near field phenomena and the photonic mode density due to the use of thin organic films. Traditional device simulation is based on the dipole model developed by Chance, Prock and Silbey (CPS) for molecular fluorescence and energy transfer near interfaces $[11,12]$. This model was extended to the near-field optics of OLEDs by various authors, [13-17], where an exciton within the OLED is modeled as a point dipole driven by the reflected electromagnetic waves inside a microcavity.

The simulations presented in this work are also based on this traditional approach of emissive dipoles. The dipoles, embedded in the multilayer stack of an OLED, are treated as forced damped harmonic oscillators $[13,14]$

$$
\text { (Eq. 1) } \quad \frac{d^{2} p}{d t^{2}}+\omega_{0}^{2} p=\frac{e^{2}}{m} E_{\mathrm{r}}-b_{0} \frac{d p}{d t}
$$

where $p$ is the electric dipole moment, $\omega_{0}$ is the resonant angular frequency in the absence of damping, $m$ is the effective mass, $e$ the electric charge, $E_{\mathrm{r}}$ is the reflected electric field at the dipole position and $b_{0}$ is the radiative decay rate in the absence of any interfaces. Both the dipole and the reflected field oscillate with the same complex frequency $\Omega=\omega-\mathrm{i} b / 2$ as:

(Eq. 2) $\quad p=p_{0} \exp (-\mathrm{i} \Omega t)$ and $E_{\mathrm{r}}=E_{0} \exp (-\mathrm{i} \Omega t)$, 
where $\omega$ and $b$ are the frequency and decay rate in the presence of interfaces. Then the modified decay rate is obtained as:

$$
b=b_{0}+\frac{e^{2}}{m \omega p_{0}} \operatorname{Im}\left(E_{0}\right) .
$$

This equation shows that the change of the decay rate inside the microcavity is determined by the magnitude of the reflected electric field at the position of the emitting dipole. It is calculated using the Hertz vector and solving the Helmholtz equation by an expansion into plane waves (for details see e.g. Lukosz et al. [18]). Thereby all layers in this multilayer stack are treated as infinite in two dimensions, having a certain thickness in the third direction. The interface between two layers is assumed sharp and without roughness. Using a transfer matrix method the Fresnel coefficients are calculated by taking the optical constants and thicknesses of the different layers of the OLED stack into account, as well as the position of the dipoles within the OLED, which is assumed to be sharp as well. The modified decay rate for arbitrary dipole orientation can then be expressed as a combination of parallel and perpendicular contributions $b_{\|}$and $b_{\perp}$, respectively, which are calculated numerically by integrating over all in-plane wave vector values of $k_{\mathrm{x}}$ from 0 to $\infty$ (for details see Chance et al. or Barnes $[12,14]$ ). Thereby the radiative quantum efficiency of the emitter (denoted as $q$ ) enters as a material parameter. Finally the orientation of the dipoles (parallel or perpendicular to the substrate, weighted $2: 1$ for isotropic dipole orientation) yielding

$$
\text { (Eq. 4) } \quad b_{\text {iso }}=\frac{2}{3} b_{\|}+\frac{1}{3} b_{\perp}
$$

and the emission spectrum of the emitter is taken into account. As a result we obtain a polychromatic power dissipation spectrum, i.e. the contribution of modes with in-plane wave vector component $k_{\mathrm{x}}$ to the spontaneous emission decay rate of the dipoles at each wavelength. (An example of such a power dissipation spectrum for a green fluorescent device calculated by this method can be found in our recent publication [8].) 
The power dissipation spectra can be split up into four regions depending on the magnitude of the in-plane wave vector component $k_{\mathrm{x}}$ as compared to the vacuum wavelength $\lambda$ and the (effective) refractive index $n$ of the medium, where the produced radiation is travelling. Region 1 with $k_{\mathrm{x}}<2 \pi n_{\text {air }} / \lambda$ represents the light which can leave the OLED directly ('direct emission'). Region 2 , where $2 \pi n_{\text {air }} / \lambda<k_{\mathrm{x}}<2 \pi n_{\text {glass }} / \lambda$ is valid, denotes the amount of light which is emitted to the glass substrate and is trapped there due to total internal reflection ('emission to substrate'). For even larger values of $k_{\mathrm{x}}$ the radiation of the dipoles does no longer reach the glass substrate but is waveguided in the organic layers and the adjacent ITO electrode ('waveguiding', region 3) with $2 \pi n_{\text {glass }} / \lambda<k_{\mathrm{x}}<2 \pi n_{\mathrm{ITO}+\mathrm{org}} / \lambda$, where $n_{\mathrm{ITO}+\text { org }}$ denotes an effective refractive index of the ITO and organic layers taken together. Finally, for $\mathrm{k}_{\mathrm{x}}>$ $2 \pi n_{\mathrm{ITO}+\mathrm{org}} / \lambda$, the emitting dipoles couple to surface plasmon polaritons (SPPs) traveling at the organic-cathode interface ('plasmons', region 4). We note that, in principle, waveguided modes and SPPs (apart from very small distances between the emitting dipoles and the metal electrodes $(\leq 25 \mathrm{~nm})$ not being relevant here) contribute to an enhancement of the spontaneous emission decay rate, although it is usually not possible to extract the energy contained in these modes [8]. Integrating over the different regions in the polychromatic power dissipation spectrum leads to the amount of power coupled into the different modes. Additionally the transmission of the generated light (i.e. 'direct emission' + 'emission to substrate') through the OLED stack is calculated from the optical constants and thickness of each layer with a transfer matrix method. Thus by comparing the internal and the transmitted power of these two modes, the total amount of light absorbed in the OLED stack is determined.

To confirm the outcome of the simulations the power dissipation spectra can be compared to the experimental angular emission spectra of the OLEDs, optionally with the distinction between s- and p-polarization. This has been done in Ref. [8] for a green bottom-emitting device and is thus not shown here. 


\section{RESULTS AND DISCUSSION}

\subsection{Effect of the radiative quantum efficiency}

In this section we discuss the impact of the radiative quantum efficiency of the emitter material $q$ on predictions of the external quantum efficiency of organic light-emitting diodes. In general the optical performance of OLEDs depends on several factors. E.g. the layer thicknesses mainly have an influence on the waveguided modes, whereas the location of the emission zone (i.e. in terms of the model: the location of the dipole layer) determines the fraction of power dissipated by the waveguided modes and the fraction coupled to surface plasmon polaritons (SPPs) [8]. Simulations of the red-emitting OLEDs described above have been performed using the model presented in section 2.2. Here the emission zone was assumed to be located at the interface between EML and HBL. This can be concluded from the excellent hole transporting properties of the host material used for the EML. Furthermore the free emitter spectrum is needed as input for the simulations. Here, we define the free emitter spectrum as the emission spectrum of the emitter in an unbounded space filled with the material(s) forming the EML. The free emitter spectrum has been extracted from the electroluminescence spectrum emitted in the direction perpendicular to the substrate by applying optical simulation on the measured data*. The computed contributions of the different loss channels as a function of ETL thickness are plotted in the graph of Fig. 4. The radiative quantum efficiency $q$ was set to be one in the calculations, i.e. the presence of processes resulting in non-radiative recombination of electrons and holes was neglected. The variation of the ETL thickness not only influences the waveguided modes (plot (2) and (5) in Fig. 4), but the dipole layer position with respect to the cathode as well. This leads to changes in the contribution of the SPP losses (6). The coupling to SPPs is reduced drastically as the

\footnotetext{
* To obtain the free emitter spectrum we first simulated the zero degree emission from the OLED using a white spectrum (constant intensity over all wavelengths) and subsequently divided the outcome by the measured ELspectrum.
} 
dipole layer moves further away from the cathode. At the same time the fraction of ITO/organic waveguided light (5) increases. Furthermore, the total amount of absorbed light (4) increases with increasing ETL-thickness. As all other layer thicknesses have been kept constant, this is mostly due to the absorbing n-dopant of the ETL. The direct emission into air (1) shows a maximum at $75 \mathrm{~nm}$ ETL thickness. The amount of light coupled from the active layers into air and substrate (3) has its maximum at $105 \mathrm{~nm}$ ETL thickness. This configuration has to be chosen if optimum efficiency based on substrate surface modification is desired. However, we have already shown in another article that the optimal dipole layer position also depends on the radiative quantum efficiency $q$ [8]. We will discuss the role of $q$ in more detail in the following.

The radiative quantum efficiency $q$ of an electroluminescent dye is defined as the quantum efficiency of electroluminescence in an unbounded space filled by the dye and its host. Competing processes resulting in non-radiative recombination of charge carriers reduce the radiative quantum efficiency. Possible competing processes include impurity quenching [19], triplet-polaron annihilation [20] or triplet-triplet annihilation [21]. The importance of the latter two is evident from the current-density dependent roll-off of the external quantum efficiency shown in figure 3.

The radiative quantum efficiency is given by:

(Eq. 5) $\quad q=k_{\mathrm{r}} /\left(k_{\mathrm{r}}+\sum k_{\mathrm{nr}}\right)$, where $k_{\mathrm{r}}$ is the radiative decay rate in an unbounded emitter material and $\Sigma k_{\mathrm{nr}}$ is the sum of the decay rates of all competing processes. In the optical cavity formed by an OLED device the radiative decay rate and the spatial distribution of the emitted radiation can be altered (see section 2.2), if a fundamental mode of the system is resonant with the free emission spectrum $[8,15]$. (Please note, that in the terminology used here, waveguided modes and SPPs are not considered as non-radiative, because the exciton is a priori generating radiation - even though it is hard to get that radiation out of the device.) Hence, the fraction of radiative decay in the 
OLED cavity can differ from the value $q$ in the unbounded emitter space. This effective fraction of radiative decay in the EML of the device is given by:

$$
q_{\mathrm{eff}}=\frac{k_{\mathrm{r}}^{*}}{k_{\mathrm{r}}^{*}+\sum k_{\mathrm{nr}}} \rightarrow q_{\mathrm{eff}}(q)=\frac{q}{q+\frac{k_{\mathrm{r}}}{k_{\mathrm{r}}^{*}}(1-q)}
$$

where $k_{\mathrm{r}}^{*}$ is the radiative decay rate determined by the boundary conditions of the electromagnetic field in the optical cavity. Such cavity effects on the radiative quantum efficiency are well explored for emitting dipoles in the vicinity of metal surfaces [11,12,22], but only rarely discussed in the context of OLEDs [16,23]. In many cases, device simulations assume $q$ to be just a multiplicative factor, which is first taken to be unity and then scaled to match the measured external quantum efficiency of a device. As we will show below, this approach leads to erroneous results when the position of the emitter in the OLED cavity is varied.

Considering the layer structure of the red-emitting OLEDs described above, $q_{\text {eff }}$ as a function of the ETL thickness is plotted for different values of the radiative quantum efficiency $q$ in Fig. 5. The effective radiative quantum efficiency $q_{\text {eff }}$ is significantly enhanced for smaller values of the ETL thickness. The enhancement is higher for lower values of the radiative quantum efficiency $q$. Fig. 6a shows the corresponding simulation results for the amount of light $\Lambda$ coupled from the active layers to the outside world (i.e. the sum of the direct emission and the substrate waveguided light). $\Lambda$ corresponds to the power fraction of all excitons generated in the emission layer which can leave the device. It can be seen, that both the values of $\Lambda$ and the progression of the maxima depend on $q$. The ETL thickness, where the maximum of light accessible by substrate surface modification is located in each curve, increases for higher values of $q$. For a given value of $q$ the location of this maximum is given by the trade-off between effective radiative rate and SPP losses, which are both dependent on the distance between the emission zone and the metal cathode (compare the curves in Fig. 5 to 
plot (6) in Fig. 4). Additionally the data shown in Fig. 6a has been normalized to the values for an ETL thickness of $40 \mathrm{~nm}$ (Fig. 6b), which was taken as reference configuration. Hence, the data of Fig. $6 \mathrm{~b}$ give the improvement of $\Lambda$ in comparison to the reference configuration. Again the achievable improvement depends on the radiative quantum efficiency $q$. Higher improvement can be obtained based on higher values of $q$, but at the same time the optimum device configuration moves to larger ETL thickness. This, however, implies that neither the optimum ETL thickness nor the achievable efficiency improvement of $\Lambda$ can be predicted based on optical simulation alone, if the radiative quantum efficiency $q$ is not known beforehand, which is usually the case for new or undisclosed emitter systems.

White-emitting OLEDs usually comprise two or more emitting layers. Consequently exact simulation based prediction of emission color (or change in emission color) of such a device is challenging without knowledge of the radiative quantum efficiencies of the individual emitting components, since the resulting white spectrum relies on the effective decay rate of the different emitters. However, optical simulation may be used to obtain the radiative quantum efficiency from experimental data as will be shown in the following section.

\subsection{Device analysis based on optical simulation}

In this section we apply the above mentioned methodology to determine both the radiative quantum efficiency and the charge recombination factor via simulation-based analysis of external quantum efficiencies of a series of devices with different ETL thickness.

The light generated by the red-emitting OLEDs and coupled from the active layers into the substrate has been measured by placing the devices equipped with a glass hemisphere into an integrating sphere. The external quantum efficiency $(E Q E)$ of this configuration is derived from the measurements of the light output and applied current:

(Eq. 7) $\quad \eta_{\mathrm{EQE}}=N_{\mathrm{ph}} / N_{\mathrm{e}}$ 
where $N_{\mathrm{ph}}$ is the number of photons emitted by the device divided by the number of electrons injected, $N_{\mathrm{e}}$. When the measured quantum efficiency is brought in correlation with simulation results, one should keep in mind that the prediction of $\Lambda$ based on our model is a power contribution. However, the color of the emission detected in the integrating sphere is almost not affected by the variation of the ETL thickness of the red-emitting devices (Table 1), i.e. there is only little redistribution of the emission spectrum. Hence, the following equation can be used to fit the measurements of the EQE as a function of ETL thickness $d$ :

$$
\eta_{\mathrm{EQE}}(d)=\gamma \cdot \eta_{\mathrm{s} / \mathrm{t}} \cdot q_{\mathrm{eff}}(q) \cdot \eta_{\mathrm{out}}=\gamma \cdot \eta_{\mathrm{s} / \mathrm{t}} \cdot \Lambda_{\mathrm{q}}(d),
$$

where $\gamma$ is the efficiency with which electrons and holes combine to form excitons (charge recombination factor), $\eta_{\mathrm{out}}$ is the light extraction efficiency, $\eta_{\mathrm{s} / \mathrm{t}}$ is the fraction of excitons that is formed in a spin state capable of radiating (for phosphorescent materials such as the redemitting dye of the considered devices this is unity) and $q_{\text {eff }}$ is the effective quantum efficiency depending on $q$ as discussed above. In principle, fitting equation (8) to the experimental thickness variation allows to extract both $q$ and $\gamma$. We note that the charge recombination factor might change with the thicknesses of the layers and applied bias, however, as we only change the thickness of the n-doped ETL, which has almost no influence on the I-V characteristics (see Fig. 2), it seems reasonable to assume the same value of $\gamma$ for all layer thicknesses.

The measured external quantum efficiency as a function of ETL thickness is plotted in Fig. 7. The devices were operated at a current density of $7.5 \mathrm{~mA} / \mathrm{cm}^{2}$ when performing the measurements in the integrating sphere. Additionally five plots of the fit-function given in Eq. 8 are shown in the same graph, where only the quantum efficiency $q$ is varied and the charge recombination factor is kept fixed as $\gamma=1$. As the EQE scales linearly with the charge recombination factor and as $\gamma$ can not be higher than one we can conclude from Fig. 7 that the quantum efficiency of the emitter must be at least $q=0.275$ or higher. 
As discussed above, $q$ determines the curve progression, i.e. the position of the maximum. Thus assuming a higher value for $q$ can lead to a better correlation with the experimental data, but requires a charge recombination factor smaller than one (see Eq. 8). This is shown in Fig. 8 for different combinations of $q$ and $\gamma$. From this figure we conclude that the quantum efficiency $q$ for this red phosphorescent emitter is about $(33 \pm 5) \%$. Still higher values of $q$ result in a mismatch of the curve progression, which can not be scaled by the linear factor $\gamma$. The best fit result (red solid line in Fig. 8) is obtained for $q=0.325$ and $\gamma=$ 0.87. For an accurate determination of both $q$ and $\gamma$, for sure, more experimental data points are required. However, our method, combining simulations and experimentally derived EQE, shows that a simultaneous determination of both quantities is possible.

Next, we confirm our method by "adjusting" a lower value of the radiative quantum efficiency. As can be seen in Fig. 3, device efficiency decreases with increasing current density. This is expected due to the enhancement of triplet-polaron and triplet-triplet annihilation at higher values of current density, i.e. the rate of competing non-radiative processes is enhanced by increasing the operational current density. The graph in Fig. 9 shows the external quantum efficiency as a function of ETL thickness as derived from the measurements of the devices operated at a high current density of $150 \mathrm{~mA} / \mathrm{cm}^{2}$. Again, first the simulation results for $\gamma=1$ are shown, yielding a lower limit of $q$ of at least 0.20 . The adaption to the curve progression is shown in Fig. 10. The best fit of the experimental data in the case of $\mathrm{j}=150 \mathrm{~mA} / \mathrm{cm}^{2}$ is obtained for $q=0.25$ and $\gamma=0.85$. (Again the value of $\gamma$ should be taken only as an estimate.) Compared to the above case this lower radiative quantum efficiency reflects the expected enhancement of non-radiative recombination at higher current density, resulting in a lower EQE (Fig. 3). Our method therefore demonstrates the ability to extract the quantum efficiency of an emitter embedded inside an OLED even for different current densities. 


\section{SUMMARY AND CONCLUSION}

In conclusion we have discussed the impact of the radiative quantum efficiency of the emitter material on predictions of the external quantum efficiency of organic light-emitting diodes. We have shown that simulation based prediction of external efficiency (or efficiency improvement) is not possible without knowledge of $q$. Moreover, not only does the value of EQE depend on this quantity, but also the optimum position of the emission zone for achieving the highest light extraction efficiency. For a given value of $q$ the location of the maximum is given by the trade-off between effective radiative rate and other losses like SPPs or waveguided modes, which are both dependent on the distance between the emission zone and the metal cathode. Furthermore, simulation based prediction of the resulting emission color (or change in emission color) of devices with several emitting layers is challenging without knowledge of the radiative quantum efficiencies of the individual emitting components.

In turn we have demonstrated that both the radiative quantum efficiency of an emitter inside an OLED stack and the charge recombination factor can be determined via simulation based analysis of experimental data. This analysis of device efficiency was successfully applied on a set of red-emitting electrophosphorescent devices.

\section{ACKNOWLEDGEMENTS}

The authors would like to thank Dr. Britta Göötz for fruitful discussion and support and Daniel Setz for processing of simulation data and performing measurements. Furthermore the authors would like to thank the German Federal Ministry of Education and Research (BMBF) for funding part of this work under contract FKZ 13N8995 (OPAL). 


\section{REFERENCES}

[1] T. Nakamura, N. Tsutsumi, N. Juni, and H. Fujii, J. Appl. Phys. 96, 6016 (2004).

[2] J.J. Shiang and A.R. Duggal, J. Appl. Phys. 95 (5), 2880 (2004).

[3] J.J. Shiang, T. Faircloth, and A.R. Duggal, J. Appl. Phys. 95 (5), 2889 (2004).

[4] C. Gärditz, R. Paetzold, D. Buchhauser, R. Bathelt, G. Gieres, C. Tschamber, A. Hunze, K. Heuser, A. Winnacker, J.R. Niklas, J. Amelung, and D. Kunze, SPIE Conf. Proc. 5937, 94105 (2005).

[5] H.J. Peng, Y.L. Ho, C.F. Qiu, M. Wong, and H.S. Kwok, SID Int. Symp. Digest, 158 (2004).

[6] M. Scheffel, A. Hunze, J. Birnstock, J. Blässing, W. Rogler, G. Wittmann, and A. Winnacker, European Conference on Organic Electronics and Related Phenomena '01, Proceedings, 158 (2001).

[7] B. Krummacher, M. Mathai, V. Choong, S.A. Choulis, F. So, and A. Winnacker, J. Appl. Phys. 100, 054702 (2006).

[8] S. Nowy, B.C. Krummacher, J. Frischeisen, N.A. Reinke and W. Brütting, J. Appl. Phys. 104, 123109 (2008).

[9] M.H. Lu. and J.C. Sturm, J. Appl. Phys. 91, 595 (2002).

[10] V. Bulovic, V.B. Khalfin, G.Gu, P.E. Burrows, D.Z. Garbuzov, and S.R. Forrest, Phys. Rev. B. 58, 3730 (1998).

[11] R.R. Chance, A. Prock, and R. Silbey, J. Chem. Phys. 60 (7), 2744-2748 (1974).

[12] R.R. Chance, A. Prock, and R. Silbey, Adv. Chem. Phys. 37, 1-65 (1978). 
[13] L. Novotny, J. Opt. Soc. Am. A 14 (1), 91-104 (1997).

[14] W.L. Barnes, J. Mod. Opt. 45 (4), 661-699 (1998).

[15] J.A.E. Wasey and W.L. Barnes, J. Mod. Opt. 47 (4), 725-741 (2000).

[16] L.H. Smith, J.A.E. Wasey, I.D.W. Samuel, and W.L. Barnes, Adv. Funct. Mat. 15 (11), 1839-1844 (2005).

[17] K. Celebi, T.D. Heidel, and M.A. Baldo, Opt. Exp. 15 (4), 1762-1772 (2007).

[18] W. Lukosz and W. Kunz, J. Opt. Soc. Am. 67, 1615 (1977).

[19] M. Pope and C.E. Swenberg, Electronic Processes in Organic Crystals and Polymers, Oxford University Press (1992).

[20] N.C. Giebink, B.W. D'Andrade, M.S. Weaver, P.B. Mackenzie, J.J. Brown, M.E. Thompson, and S.R. Forrest, J. Appl. Phys. 103, 044509 (2008).

[21] M.A. Baldo, C. Adachi, and S.R. Forrest, Phys. Rev. B 62, 10967-10977 (2000).

[22] K.H. Drexhage, Progress in Optics 12, 163-232 (1974).

[23] H. Becker, S.E. Burns, and R.H. Friend, Phys. Rev. B 56, 1893-1905 (1997). 


\section{FIGURES}

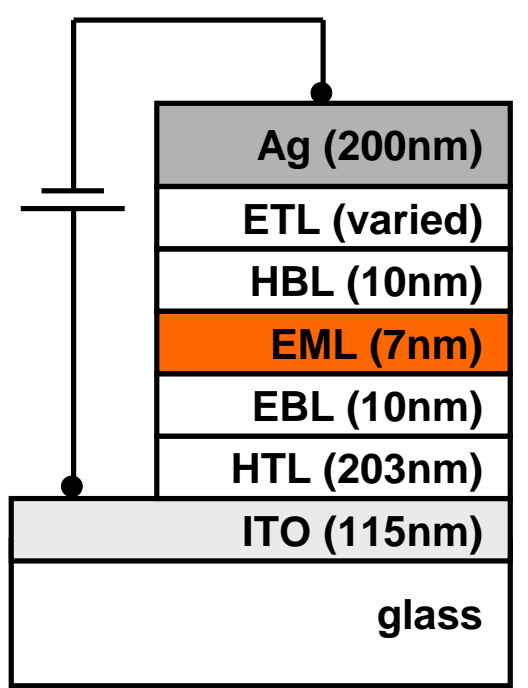

Fig. 1. Structure of the red-emitting electrophosphorescent OLEDs used in this study. The thickness of the ETL has been varied in the range between $48 \mathrm{~nm}$ and $110 \mathrm{~nm}$. 


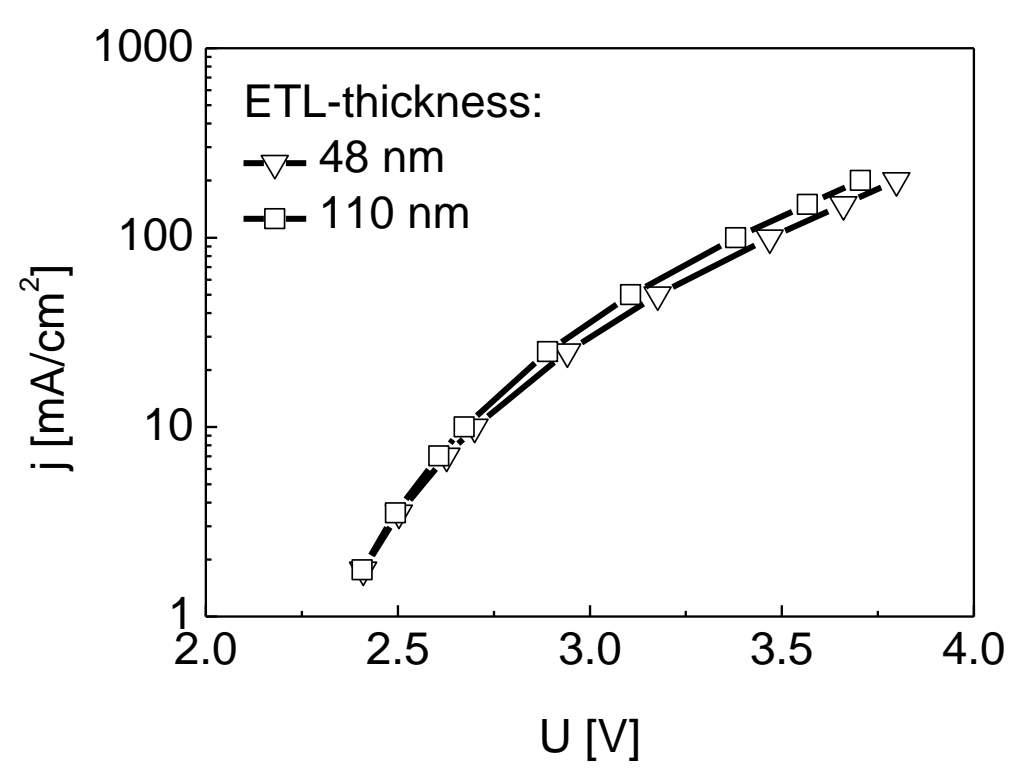

Fig. 2. Current density vs. voltage characteristics of devices with ETL thickness of $48 \mathrm{~nm}$ and $110 \mathrm{~nm}$. 


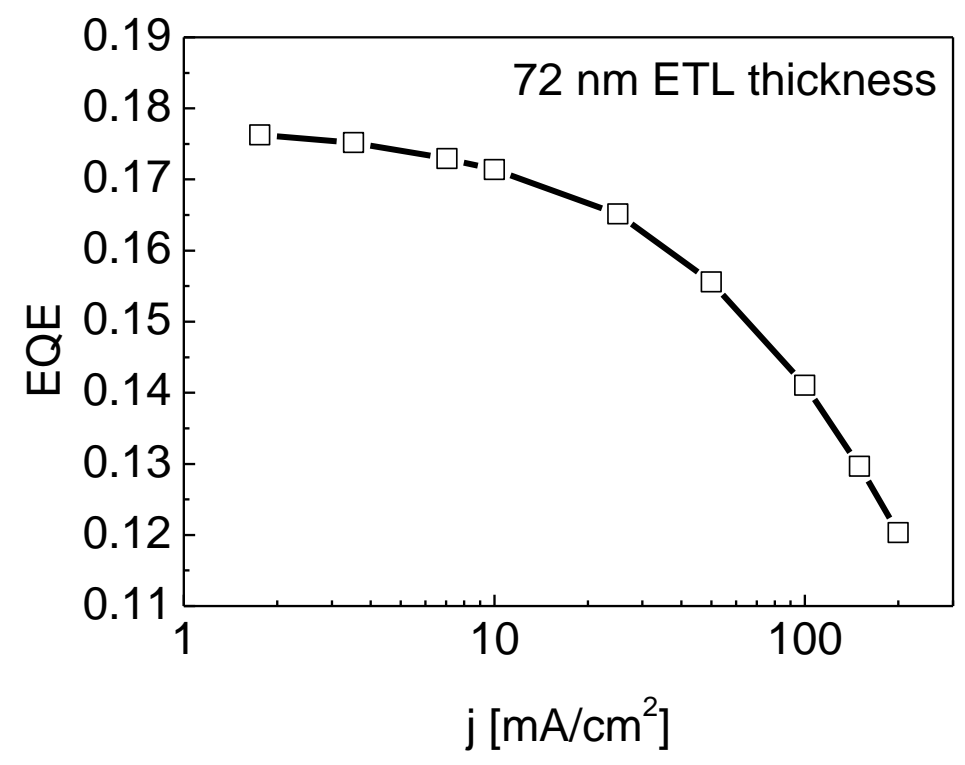

Fig. 3. Plot of the measured external quantum efficiency as a function of current density for the device with an ETL-thickness of $72 \mathrm{~nm}$. 


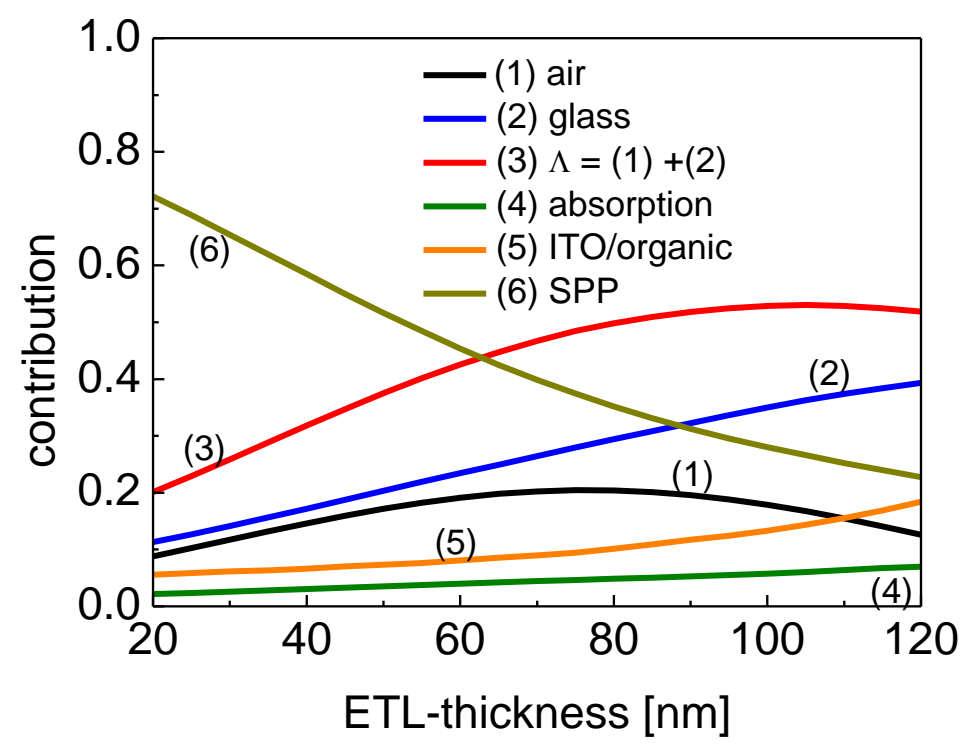

Fig. 4. Plots of the relative contributions of the different optical loss channels as a function of the ETL thickness. The data was obtained from simulation, where the radiative quantum efficiency was set to $q=1$. 


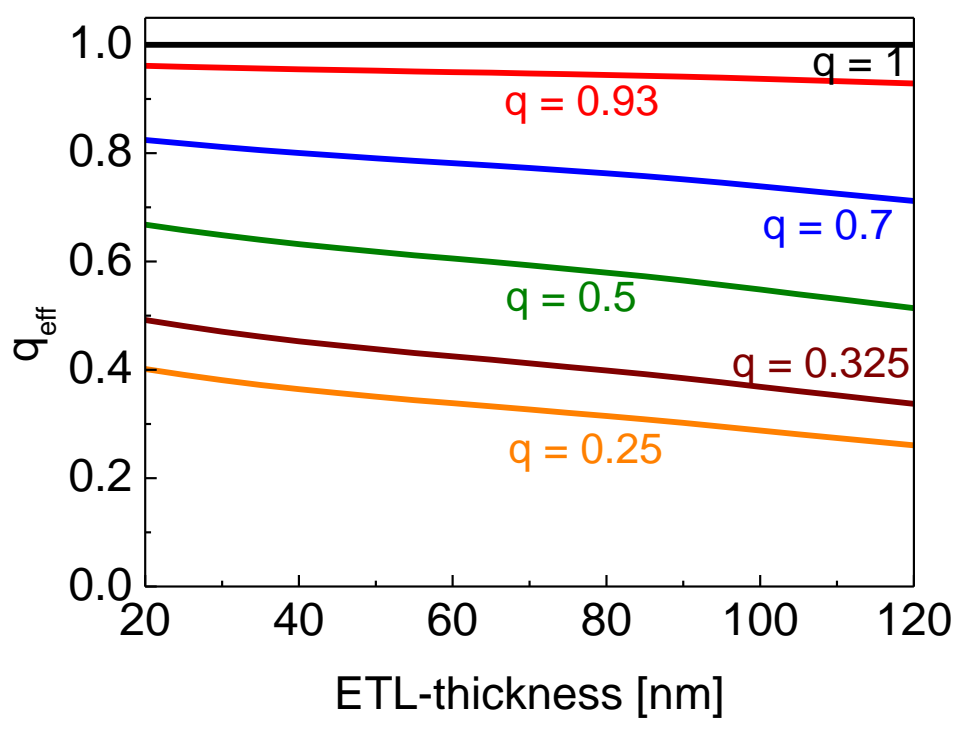

Fig. 5. Effective fraction of radiative decay in the emission layer as a function of layer thickness for different values of the radiative quantum efficiency $q$. 

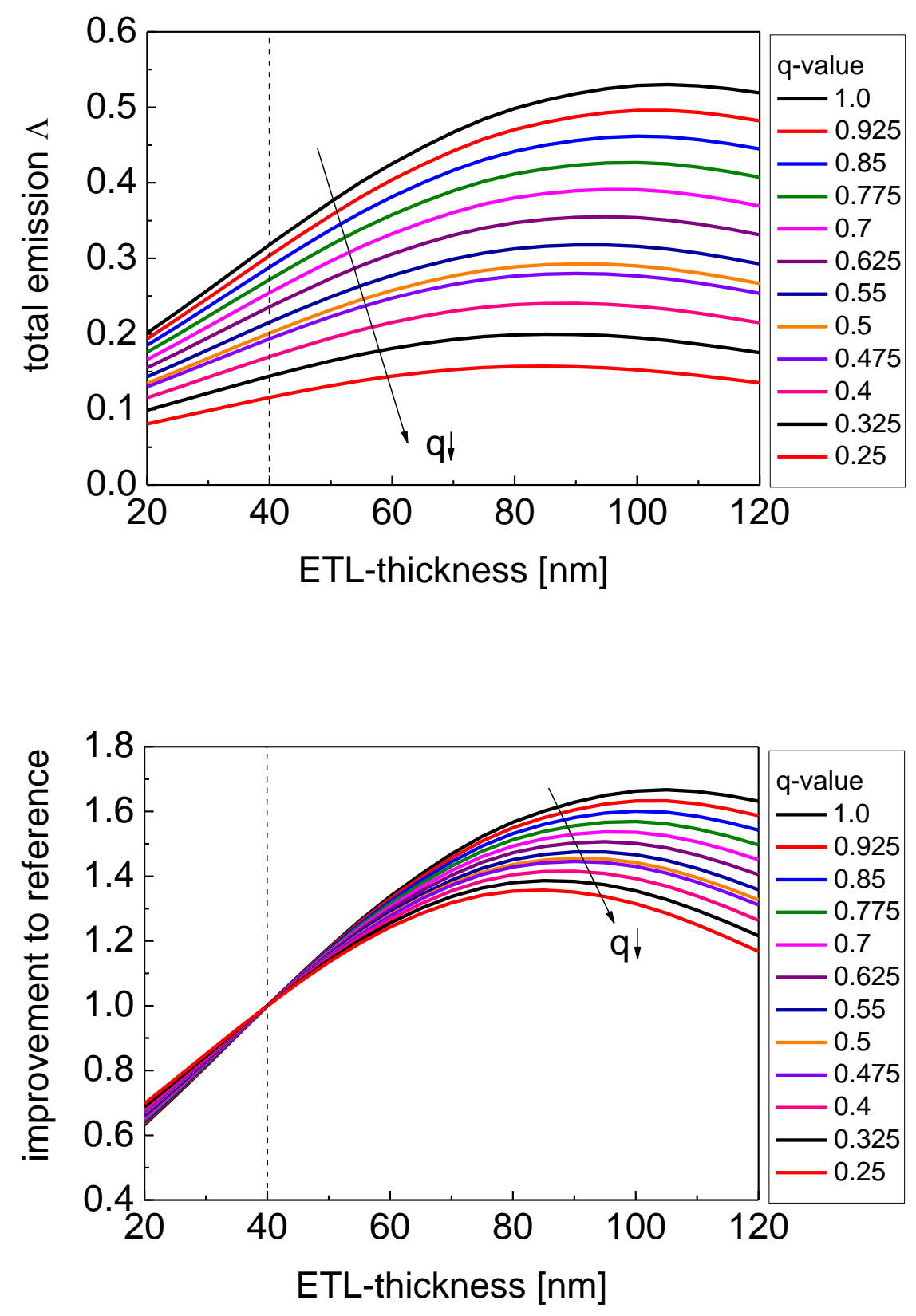

Fig. 6. (a) Plot of the generated light coupled from the active layers to the outside world (total emission $\Lambda=$ air + glass). The data was obtained by simulation assuming different values of q. (b) $\Lambda$ as a function of ETL thickness normalized on the values at $40 \mathrm{~nm}$ ETL thickness. The plot gives the efficiency improvement in comparison to the reference device with $40 \mathrm{~nm}$ ETL. 


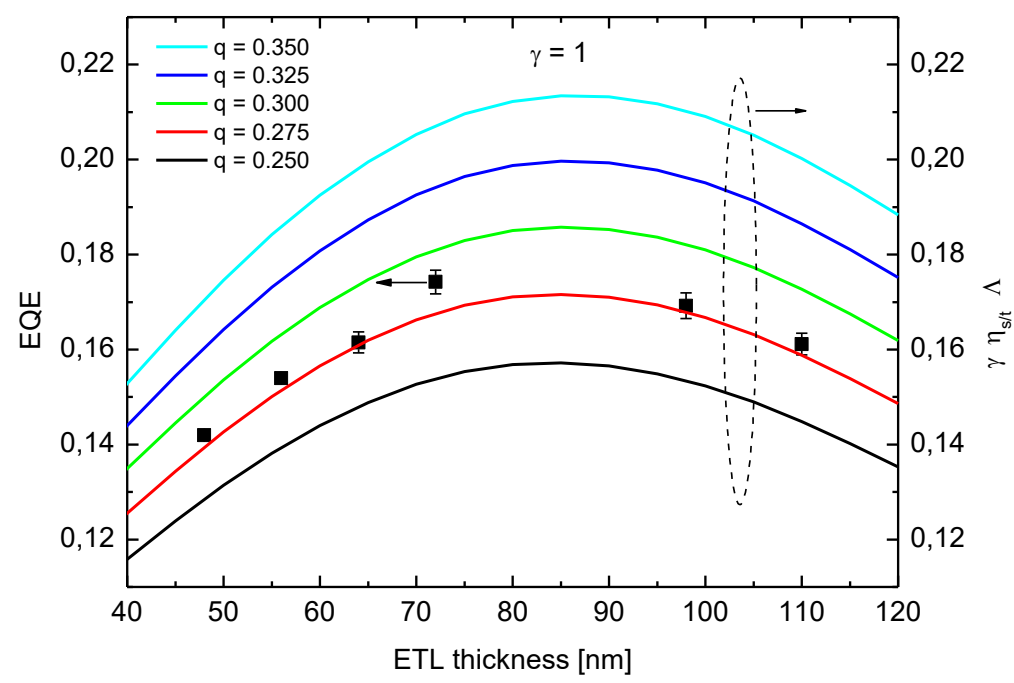

Fig. 7. Black squares: measured external quantum efficiency as a function of ETL thickness (device operation at current density of $7.5 \mathrm{~mA} / \mathrm{cm}^{2}$ ). The error bars represent the standard deviation resulting from the measurements of several devices with the same ETL thickness. Solid lines: corresponding simulations for different quantum efficiencies $q$ with a fixed charge recombination factor $\gamma=1$. 


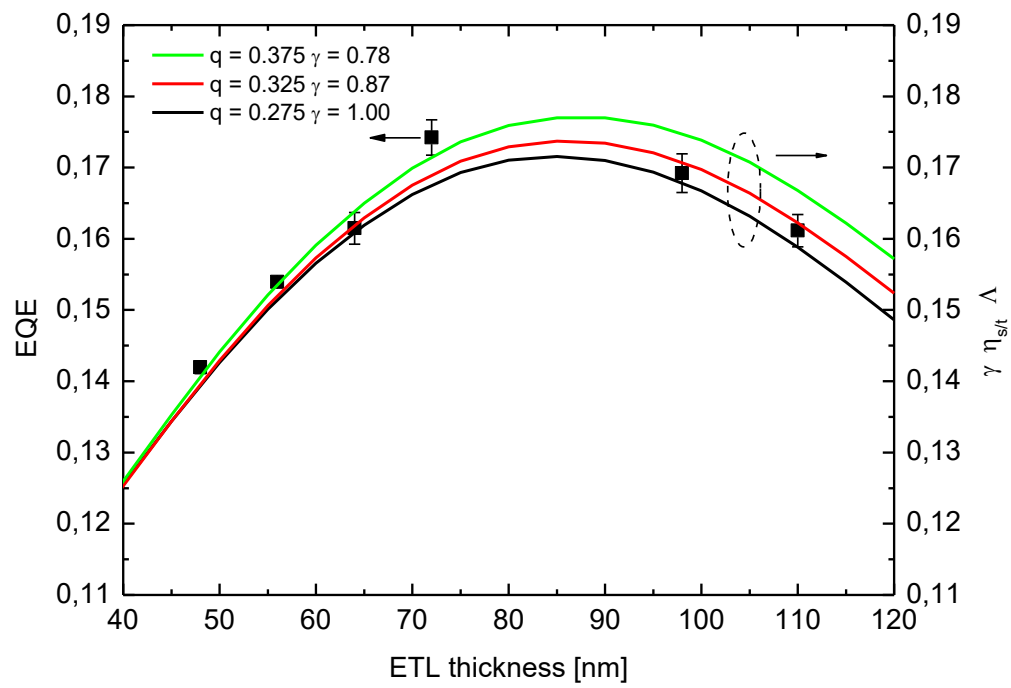

Fig. 8. Black squares: measured external quantum efficiency as a function of ETL thickness (same data as in Fig. 7). Solid lines: corresponding simulations for different combinations of quantum efficiency $q$ and charge recombination factor $\gamma$ (see Eq. 8). 


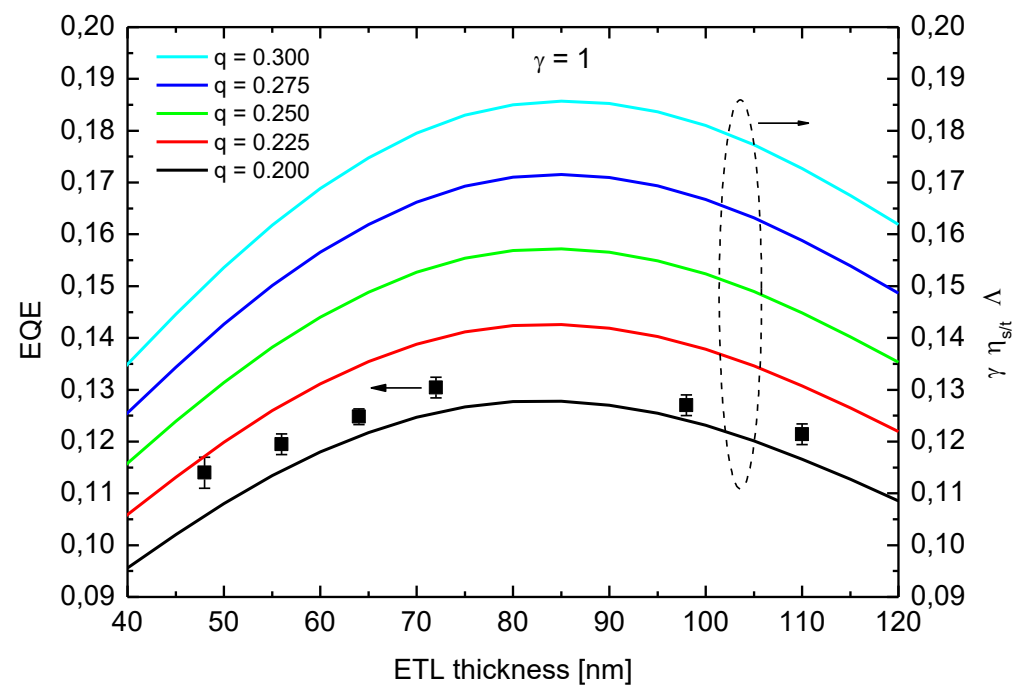

Fig. 9. Black squares: measured external quantum efficiency as a function of ETL thickness (device operation at current density of $150 \mathrm{~mA} / \mathrm{cm}^{2}$ ). Solid lines: corresponding simulations for different quantum efficiencies $q$ with a fixed charge recombination factor $\gamma=1$. 


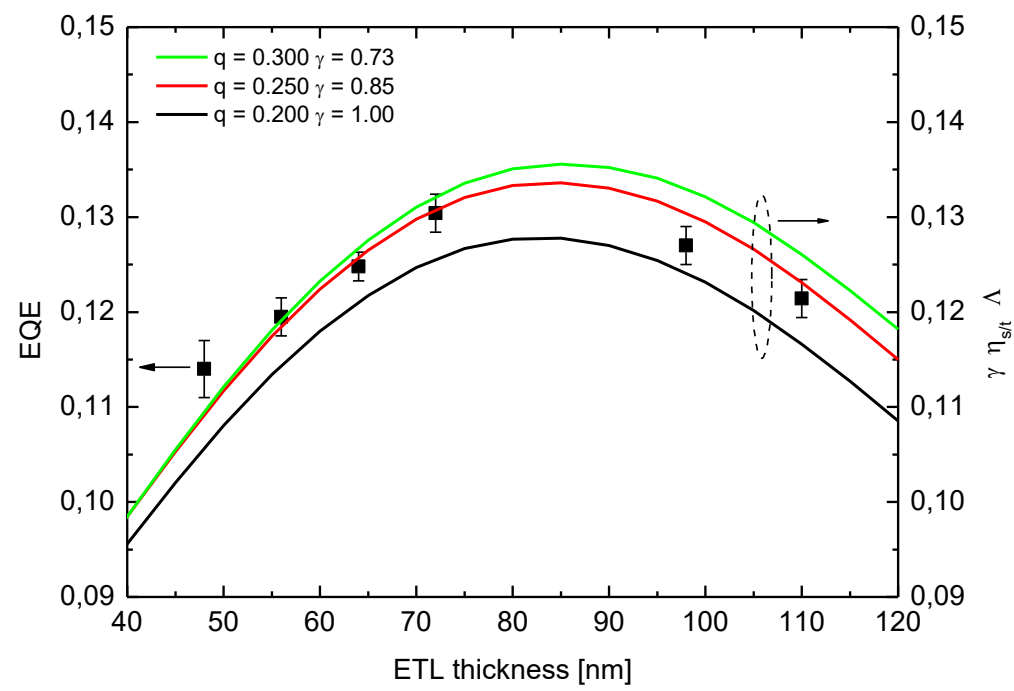

Fig. 10. Black squares: measured external quantum efficiency as a function of ETL thickness (same data as in Fig. 9). Solid lines: corresponding simulations for different combinations of quantum efficiency $q$ and charge recombination factor $\gamma$ (see Eq. 8). 


\section{TABLES}

Table 1. CIE color coordinates related to the emission measured in an integrating sphere. The devices were operated at a current density of $7.5 \mathrm{~mA} / \mathrm{cm}^{2}$.

\begin{tabular}{|c|c|c|} 
ETL-thickness & CIE x & CIE y \\
\hline \hline $48 \mathrm{~nm}$ & 0.612 & 0.387 \\
$56 \mathrm{~nm}$ & 0.612 & 0.386 \\
$64 \mathrm{~nm}$ & 0.613 & 0.385 \\
$72 \mathrm{~nm}$ & 0.614 & 0.384 \\
$98 \mathrm{~nm}$ & 0.616 & 0.383 \\
$110 \mathrm{~nm}$ & 0.617 & 0.380 \\
\hline \hline
\end{tabular}

